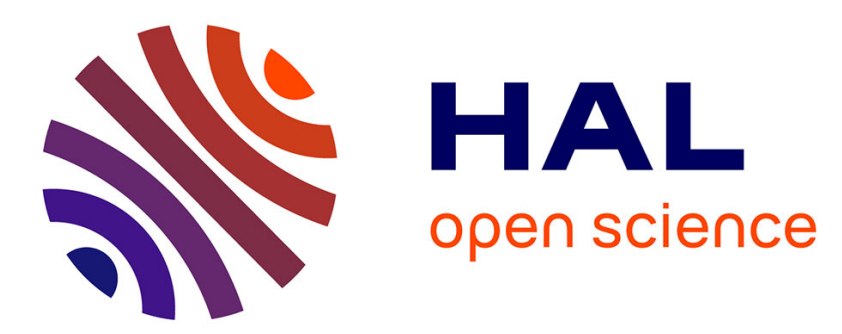

\title{
Ionic liquid-based organically modified silica for the development of new electrorheological fluids
}

\author{
Jéssica Alves Marins, Bluma G Soares
}

\section{To cite this version:}

Jéssica Alves Marins, Bluma G Soares. Ionic liquid-based organically modified silica for the development of new electrorheological fluids. Colloids and Surfaces A: Physicochemical and Engineering Aspects, 2017, 529, pp.311-319. 10.1016/j.colsurfa.2017.05.091 . hal-01674667

\section{HAL Id: hal-01674667 \\ https://hal.science/hal-01674667}

Submitted on 3 Jan 2018

HAL is a multi-disciplinary open access archive for the deposit and dissemination of scientific research documents, whether they are published or not. The documents may come from teaching and research institutions in France or abroad, or from public or private research centers.
L'archive ouverte pluridisciplinaire $\mathbf{H A L}$, est destinée au dépôt et à la diffusion de documents scientifiques de niveau recherche, publiés ou non, émanant des établissements d'enseignement et de recherche français ou étrangers, des laboratoires publics ou privés. 


\title{
Ionic liquid-Based Organically Modified Silica for the Development of new Electrorheological Fluids
}

\author{
Jéssica A. Marins ${ }^{1-2^{*}}$ and Bluma G. Soares ${ }^{2}$ \\ ${ }^{1}$ Université Côte d'Azur, UMR 7010 Institute of Physics of Nice, Parc Valrose 06100 \\ Nice, France \\ ${ }^{2}$ Universidade Federal do Rio de Janeiro, Departamento de Engenharia Metalúrgica e de \\ Materiais, Centro de Tecnologia, Bl. F, 21941-972, Rio de Janeiro, RJ, Brazil;
}

\begin{abstract}
This work describes the preparation of novel electrorheological (ER) fluids consisted of organically modified silica particles with imidazolium-based ionic liquid (ILORMOSIL) suspended in a silicone oil. The IL-ORMOSIL was prepared by hydrolysis/co-condensation of tetraethoxy silane (TEOS) in the presence of different concentration of 1-(3-trimethoxysilylpropyl)-3-methylimidazolium chloride (ILTMOS), using the one-pot sol-gel process. IL-ORMOSIL particles with controlled morphology were obtained, as indicated by transmission electronic microscopy (TEM). The presence of the silylated ionic liquid covalently attached to the silica particles was confirmed by thermogravimetric analysis (TGA), Fourier transform infrared spectroscopy (FTIR) and X-ray photoelectron spectroscopy (XPS). The effect of the amount of imidazolium-based ionic liquid immobilized on the spherical silica particles on the electrorheological (ER) behavior of the corresponding suspensions in silicone oil was investigated. A significant ER effect was observed for the fluid containing silica modified with around 10\%wt. of IL-TMOS. In fact, a very good, extremely rapid and reversible response under the action of an electric field at intensity to $4 \mathrm{kV} / \mathrm{mm}$ was achieved, with shear stress value as high as $2200 \mathrm{~Pa}$. The dielectric properties were also evaluated to explain the ER response of these IL-ORMOSIL particles
\end{abstract}

Keywords: ionic liquid; ORMOSIL; sol-gel process; electrorheological fluids; dielectric properties.

\section{Introduction}


Electrorheological fluids (ERF) belong to a class of smart materials which exhibit sharp and reversible phase transition from a viscous fluid-state to a solidlike state under an applied external electric field [1,2]. Such fluids consist of electrically polarizable particles randomly suspended in an insulating oil. As an external electric field is applied, such particles become polarized and aligned along the electric field direction and can attract each other by strong dipole-dipole interactions. As a consequence, fibril-like structures are formed in the medium, thus contributing to a drastic change in the rheological properties of the ERF $[3,4]$. Due to these unique characteristics, ERFs are considered as smart materials and have been experienced a great interest in several engineering applications, such as damping devices, shock absorbers, torque transducers, as well as human muscle stimulators, etc. The outstanding electrorheological (ER) performance of these fluids strongly depends on the dielectric properties and polarizability of the suspended particles, its volume fraction, shape, size and morphology. Among several particles, silica has been widely studied because of its low cost, relatively good dielectric properties and feasibility of preparation with tuned morphology (size and shape), by using the so-called sol-gel process. Indeed, several works have discussed the ER behavior of fluids containing silica [3,5-7], and mesoporous silica [8-11]. Several strategies have also been adopted to improve the dielectric properties and polarizability of silica particles, including incorporation of small, such molecules as water [6], urea [12-13], as well as some cation ions [6,14-15]. Another interesting approach involves the use of organically modified silica (ORMOSIL) to develop stable dispersions and increase the polarizability. In this context, silica bearing amino- [9], cyano- [9], and more recently mercapto groups [16] were used for the development of ERF. Such particles are characterized by core-shell structure consisted by silica as the core and a polarizable group as the shell. The concept of core-shell structured particles for ERF has been also applied in other systems, including polymeric as core coated with conducting polymers [17-18].

Recently, the use of ionic liquid (IL) for the preparation of silica particles has been emerged as a new and promising technology for the development of the socalled ionogels [19-25]. Due to the unique characteristics of IL such as excellent thermal stability, good ionic conductivity and negligible vapor pressure, such ionogels containing IL confined within the silica particles are expected to 
promote good ER response of ERF. Indeed, our previous work described the preparation of silica in the presence of triphenyl-phosphonium-based IL and studied the effecticiency of these ionogels used for preparation of ERF [26]. The use of $10 \%$ of such silica led to reasonable ER performance. Also, some alkyl phosphonium-based IL have recently been used on the sol-gel synthesis of silica [27]. The presence of confined IL resulted in an increase of dielectric permittivity and also in an outstanding ER effect. A drastic and reversible increase of the shear stress under the action of an electric field of $3 \mathrm{kV} / \mathrm{m}$ was observed for the ERF containing $30 \%$ of IL-confined silica. However, IL physically adsorbed on the silica may present some leaching, thus losing the dielectric properties with time. To avoid these drawbacks the IL should be immobilized within the support through covalent bonding. This strategy has been reported, using polyionic liquid particles [28-29]. However, to the best of our knowledge, the use of ionic liquid covalently linked to the silica particles for the development of ERF has never been reported.

The preparation of IL-based ORMOSIL has been performed through the coupling reaction of IL bearing silane groups on the surface of pre-formed silica nanoparticles [30]. Such IL-based ORMOSIL has been employed as sorbent for solid phase extraction [31], catalysis [32-33], electrochemical sensors [34], and as a component for the development of dye-sensitized solar cells [35].

Another approach for the development of this kind of IL-ORMOSIL has recently been reported by our group and consisted of performing the hydrolysis/co-condensation of tetraethoxy-silane (TEOS) together with trialkoxysilane containing IL [36]. These composite were used as reinforced fillers for epoxy networks.

The aim of the present work is to prove the feasibility of using silica bearing covalently bonded IL as the polarizable particles for ERF. For this purpose, silica bearing different amounts of imidazolium-based IL was first prepared by one pot sol-gel process involving the hydrolysis/co-condensation of TEOS and the ILsilane precursor followed by its dispersion/re-suspension in silicone oil. The ER activity of the resulting fluids was evaluated under different shear rates and electric field strengths. Additionally, the on-off test (allowing to follow the rheological response of ERF upon periode switching on and off the electric field) was performed in order to analyze the reversibility of the ER reponse. 
Furthermore, the dielectric properties of the fluids were also investigated and related to the ER response of the fluids.

\section{Materials and methods}

\subsection{Materials}

Tetraethoxysilane (TEOS), ammonium hydroxide $\left(\mathrm{NH}_{4} \mathrm{OH}\right)$, 1-methylimidazole, 3 chloropropyl-trimethoxysilane (CPTMS) and absolute ethanol were purchased from Sigma Aldrich. Silicone oil was purchased from Epoxy Fiber (Brazil) (Rhodorsil 47V 500) and presents the following characteristics: kinematic viscosity of $475-525 \times 10^{-6} \mathrm{~m}^{2} \cdot \mathrm{s}^{-1}$; density of around $0.97 \mathrm{~g} . \mathrm{cm}^{-3}$; conductivity of 1 $\mathrm{x} 10^{-12}{\mathrm{~S} . \mathrm{cm}^{-1}}$ at $25^{\circ} \mathrm{C}$.

\subsection{Preparation of IL-modified silica (IL-ORMOSIL).}

The synthesis of the silylated imidazolium-based IL precursor (IL-TMOS) was performed according to the protocol described in the literature [36-37]. The sketch of the synthesis is illustrated in Figure 1. The silica and IL-ORMOSIL were prepared according to the Stöbe method using the sol-gel procedure [38], and adapted from our previous work [36]. In a typical procedure, $20 \mathrm{~mL}(0.09$ mol) of TEOS was slowly added to a mechanically stirred (600 rpm) solution containing $70 \mathrm{~mL}$ of ethanol, $9 \mathrm{~mL}$ of de-ionized water and $6 \mathrm{~mL}$ of ammonium hydroxide, at room temperature. After 20 minutes of the TEOS addition, different amounts of the IL-TMOS precursor solubilized in $10 \mathrm{~mL}$ of ethanol was added. The medium was kept under stirring at room temperature for 4 hours. The white precipitate was obtained by centrifugation (16000 rpm for 30 minutes), followed by washing with water and ethanol four times, and dried at $100^{\circ} \mathrm{C}$ for 24 hours. Pure silica was prepared similarly. The IL-ORMOSIL prepared with 5, 10 and 20 wt\% of IL-TMOS was denoted as IL-ORMOSIL 1, IL-ORMOSIL 2 and ILORMOSIL 3, respectively. 


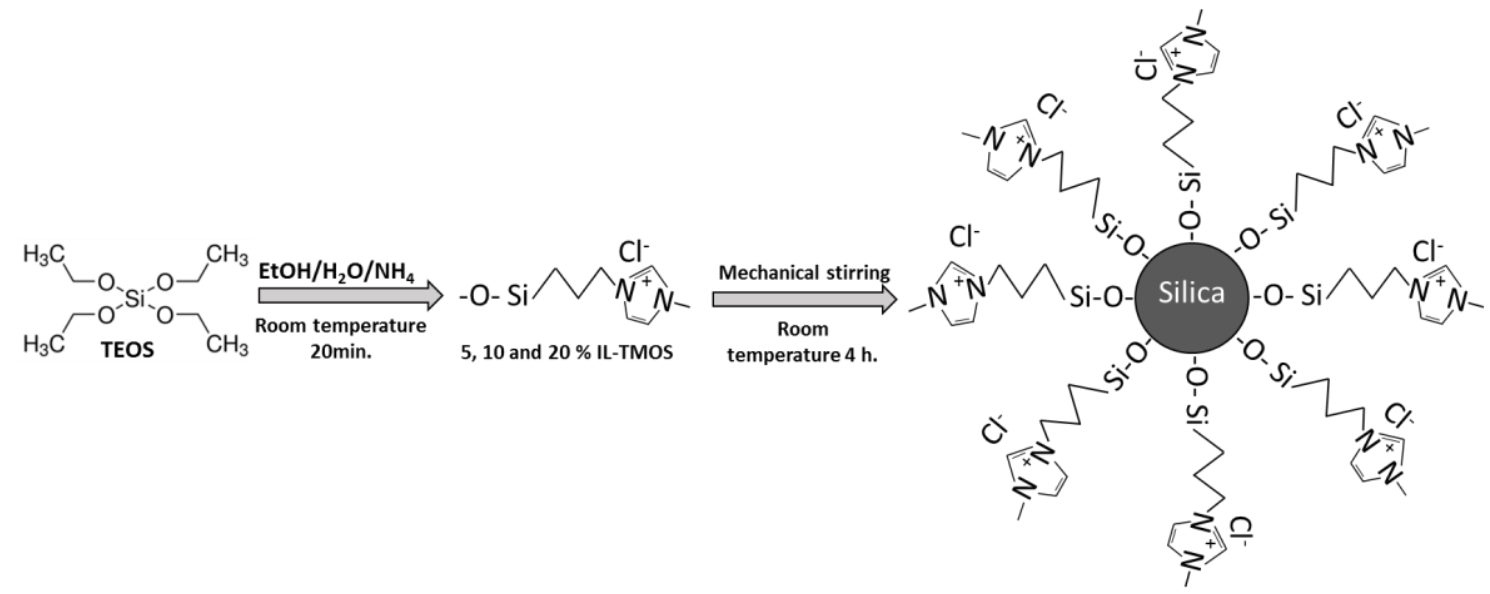

Figure 1. Synthesis of silica particles followed by immobilization of silane-ionic liquid (ORMOSIL)

\subsection{Preparation of the electrorheological fluids.}

The suspensions for ERF and dielectric measurements were prepared by dispersing silica and the corresponding IL-ORMOSIL (15 vol\%) in silicone oil, with the help of an ultrasonic apparatus (Sonifier®S-450D digital Branson equipment) operating at a power of $90 \mathrm{~W}$ for $5 \mathrm{~min}$, at $10^{\circ} \mathrm{C}$ this suspensions were stored in a desiccator prior to use.

\subsection{Characterization.}

Thermogravimetric analysis was performed in a TA Instruments Q50 V20.10 Build 36 analyzer operating at a heating rate of $20^{\circ} \mathrm{C} / \mathrm{min}$, under nitrogen atmosphere (balance gas: nitrogen $40.0 \mathrm{~mL} / \mathrm{min}$ and sample gas: nitrogen 60.0 $\mathrm{mL} / \mathrm{min}$ ). The TGA profiles were recorded in the temperature range $25-700^{\circ} \mathrm{C}$. The weight of the sample used was about 9-11 $\mathrm{mg}$ in all cases.

Fourier transformed infrared spectra (FTIR) were recorded from $\mathrm{KBr}$ pressed discs in a Nicolet Thermo Scientific, Model iS-50 Spectrometer in the range of wavenumbers of $4000-400 \mathrm{~cm}^{-1}$ with 32 scans. The sample $(\sim 1 \mathrm{mg})$ and $\mathrm{KBr}$ ( $~ 99 \mathrm{mg})$ were ground together in an agate mortar until the sample is well dispersed.

X-ray photoelectron spectroscopy (XPS) analysis was performed using an Escalab $250 \mathrm{Xi}$ electron spectrometer from Thermo Scientific equipped with a hemispherical electron energy analyzer and Al KaX-ray source (1486.6 eV), located at the Laboratório de Espectroscopia de Fotoelétrons/ Instituto de 
Química (UFRJ). The analyzer was operated in constant analyzer energy (CAE) mode.

The morphology of the silica particles was evaluated by transmission electron microscopy (TEM) (ZEISS10A) operating at $80 \mathrm{kV}$. The samples were previously deposited onto the grids with a thin film of pure carbon deposited on one side of the grid.

The density of the particles was determined on a Helium Gas Pycnometer, model AccuPyc II 1340.

The ER properties of the fluids were measured with the help of Anton Paar Instruments Physical MCR 302 rheometer equipped with plate-plate geometry (PP50/E gap $1 \mathrm{~mm}$ ) and an electrorheological unit (HVS/ERD80-DC) with a high voltage generator $(10 \mathrm{kV}, 1 \mathrm{~mA})$.

Dielectric properties of the fluids were measured using a Discovery HR-1 Hybrid Rheometer from TA Instrument, Inc., equipped with DHR dielectric unit and an Agilent E4980A LCR Meter, operating from $100 \mathrm{~Hz}$ to $1 \mathrm{MHz}$ and a voltage of $20 \mathrm{~V}$.

\section{Results and Discussion}

\subsection{Material characteristics.}

The TEM micrographs of silica and the IL-ORMOSILs are illustrated in Figure 2. Pure silica particles presented a smooth surface and spherical morphology with diameter of about $320 \mathrm{~nm}$. The IL-ORMOSIL particles displayed similar particle size but formed the aggregates. Moreover, several smaller particles of around $30 \mathrm{~nm}$ in diameter can be observed as the amount of IL-TMOS increases during the synthesis, characterizing a hierarchical structure. In our previous work related to the synthesis of similar IL-ORMOSIL, gel was obtained after a few minutes of sol-gel reaction [36]. The differences in the morphological characteristics reported in both works can be attributed to the following experimental procedures used in the present work: (i) the later addition of the IL-TMOS precursor (after the transparent solution became translucide and (ii) the faster stirring process (mechanical stirring), which minimizes or prevents the inter-particles condensation and the gel formation. Therefore, the IL-TMOS precursor should condense at the silica surface, allowing the grafting without 
changing the shape of the silicon network formed during the hydrolysiscondensation of TEOS. The procedure used in this work is interesting as it enables the synthesis of silica particles and immobilization of the IL-TMOS precursor in one-pot step. The morphology with a rough surface is very interesting for ERF because it gives stronger ER effect compared to a smooth surface [16,39-40].

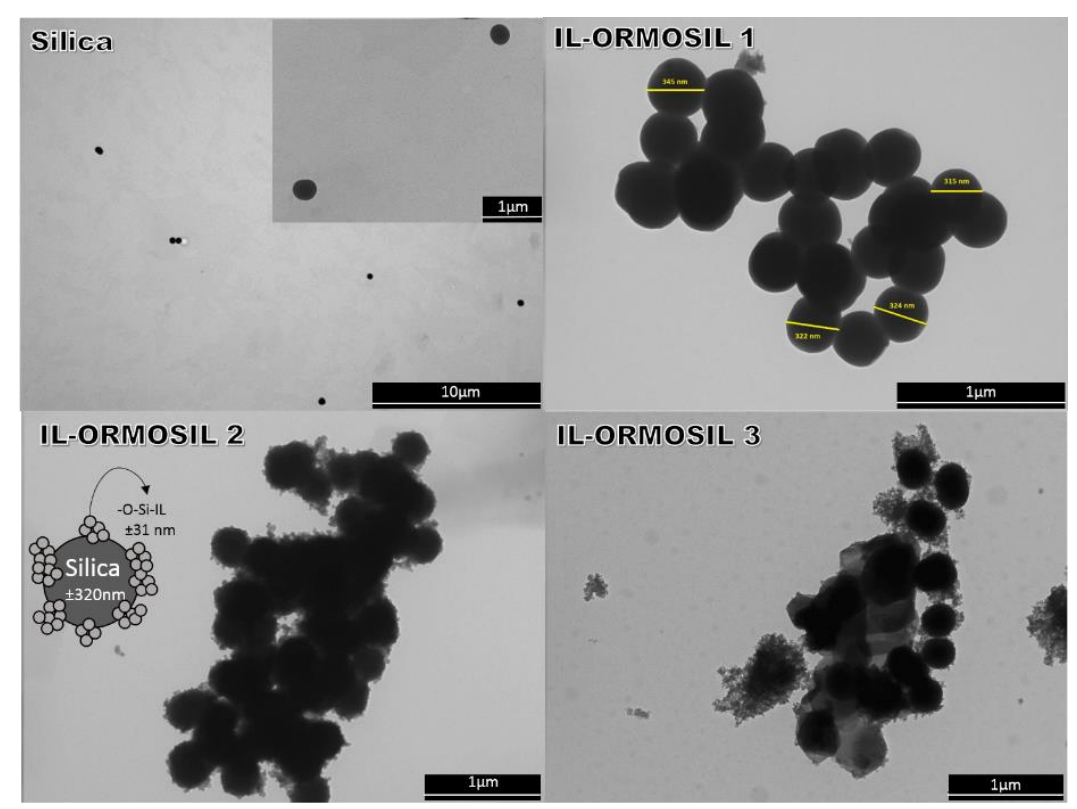

Figure 2. TEM of silica, and the IL-ORMOSILs prepared with 5\% (ILORMOSIL 1), $10 \%$ (IL-ORMOSIL 2) and 20\% (IL-ORMOSIL 3) of IL-TMOS precursor.

The presence of silylated IL covalently linked to the IL-ORMOSIL particles was suggested by FTIR spectroscopy. Figure 3 compares the FTIR spectrum of the IL-TMOS precursor with those obtained from the IL-ORMOSL and silica particles. The spectrum of the IL-TMOS precursor (Figure 3A) displayed the following absorption bands: at $1080 \mathrm{~cm}^{-1}$ assigned to the $\mathrm{Si}-\mathrm{O}$ stretching; at 924 and $816 \mathrm{~cm}^{-1}$ assigned to the $\mathrm{Si}-\mathrm{O}-\mathrm{C}$ symmetric stretching vibration; at 3125 and $3085 \mathrm{~cm}^{-1}$ attributed to stretching vibration of aromatic imidazole rings; at 2946 and $2841 \mathrm{~cm}^{-1}$ corresponding to the $\mathrm{C}-\mathrm{H}$ stretching vibration of alkyl and methyl groups, respectively; at 1571 and $1457 \mathrm{~cm}^{-1}$ assigned to the $\mathrm{C}=\mathrm{C}$ stretching vibration of imidazole ring and $\mathrm{C}-\mathrm{H}$ bending vibration of alkyl groups, respectively, and also the band at 1639 and $1184 \mathrm{~cm}^{-1}$ assigned to the $\mathrm{C}=\mathrm{N}$ 
stretching vibration and $\mathrm{C}-\mathrm{N}$ of the imidazolium ring, respectively [37]. These bands successfully confirm the synthesis of the IL-TMOS precursor.

The FTIR spectra of the silica and IL-ORMOSIL particles are shown in the Figures $3 \mathrm{~B}$ and $3 \mathrm{C}$. The broad band centered at $3428 \mathrm{~cm}^{-1}$ is related to the stretching vibration of $-\mathrm{OH}$ groups on silanol moieties and adsorbed $\mathrm{H}_{2} \mathrm{O}$ molecules. This large band makes it difficult to clearly visualize the absorption band related to the imidazole ring and the bands assigned to the stretching vibration of $\mathrm{C}-\mathrm{H}$. However it is possible to observe the small bands 3158, 2984 and $2922 \mathrm{~cm}^{-1}$ present in the IL-ORMOSIL, which increases as the amount of incorporated IL-TMOS precursor in the sample increases. The effective incorporation of the IL-TMOS into the silica particles is confirmed by the presence of the bands centered at $1573 \mathrm{~cm}^{-1}$ assigned to the imidazolium ring $[37,41]$, and at $1167 \mathrm{~cm}^{-1}$, attributed to the changes in Si-O-Si bonding angles due to the presence of the silsesquioxane moiety [42].
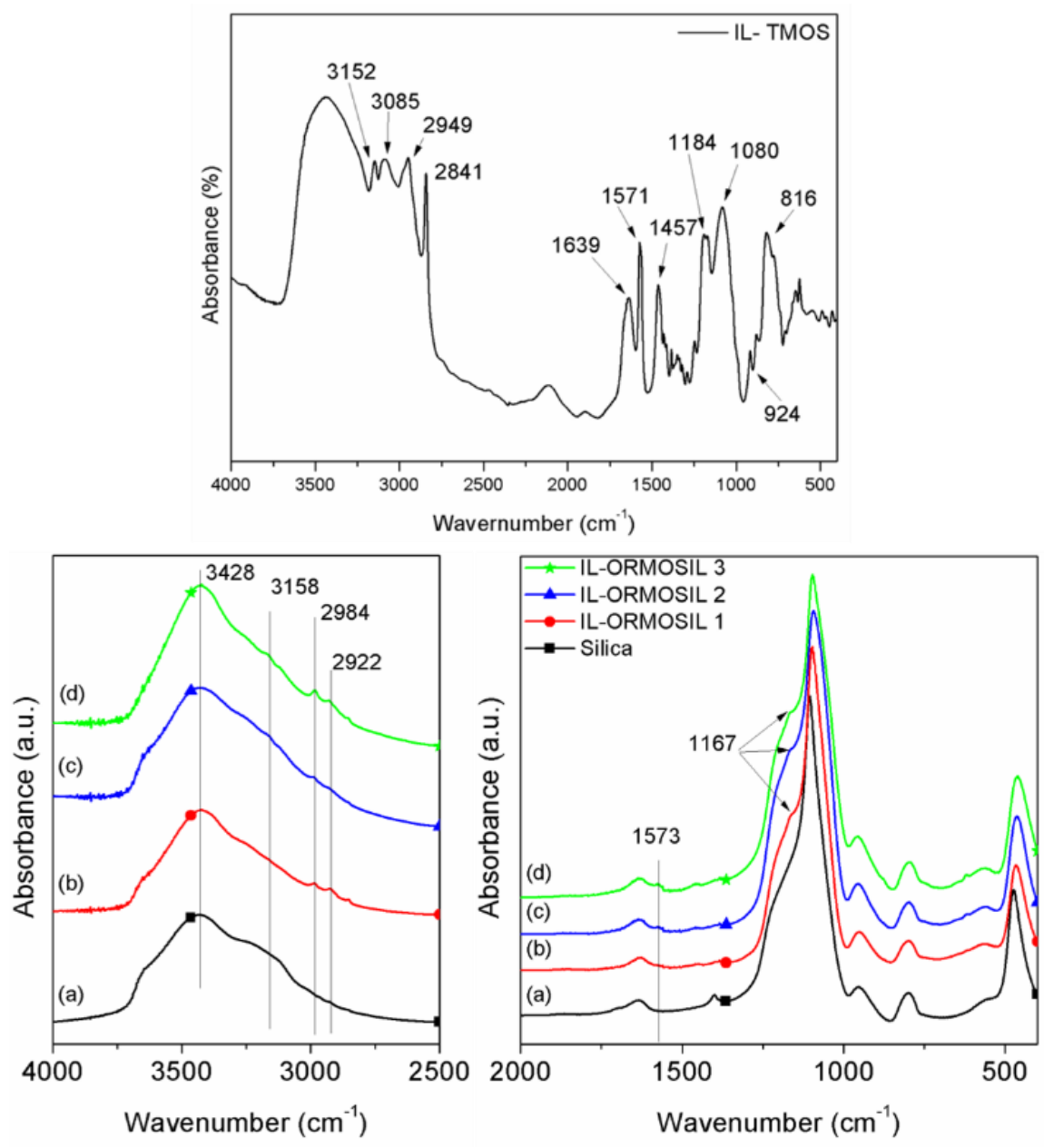

Figure 3. FTIR spectra of pure silane-ionic liquid (A) and FTIR spectra (4000 - 2500 
and $2000-400 \mathrm{~cm}^{-1}$ ) of pure silica particles (a), and the IL-ORMOSIL prepared with 5\% (IL-ORMOSIL 1), 10\% (IL-ORMOSIL 2) and 20\% (IL-ORMOSIL 3) of IL-TMOS precursor

TGA analysis was employed to evaluate the thermal stability of the ILORMOSIL samples and also to estimate the amount of IL-TMOS precursor grafted onto the silica particles. Figure 4 compares the TGA analysis of pure silica and the IL-ORMOSIL samples with different concentration of the silane precursor. All samples presented the first decomposition step below $200^{\circ} \mathrm{C}$, assigned to the adsorbed water and other volatile compounds present in the systems. Pure silica also displayed a second step of decomposition in the range of $200-700{ }^{\circ} \mathrm{C}$, which is attributed to slow dehydroxylation of internal and surface $\mathrm{OH}$ silanol groups [43]. Regarding the ORMOSIL, the second and third decomposition peaks at around $310^{\circ} \mathrm{C}$ and $500^{\circ} \mathrm{C}$ corresponded to the IL moiety attached to the silica particle [36,44].

The TGA technique allows estimating the proportion of the IL-TMOS incorporated into silica particles. For this calculation, the mass loss between 200 and $700^{\circ} \mathrm{C}$ in the IL-ORMOSIL samples was compared to that obtained for pure silica, whose results are summarized in Table 1. From this calculation, it was possible to estimate an amount of incorporated IL of around 2.9, 8.5 and $13.9 \%$ for the IL-ORMOSIL 1, IL-ORMOSIL 2 and IL-ORMOSIL 3, respectively, so over $50 \%$ of ionic liquid was incorporated into the silica particles.

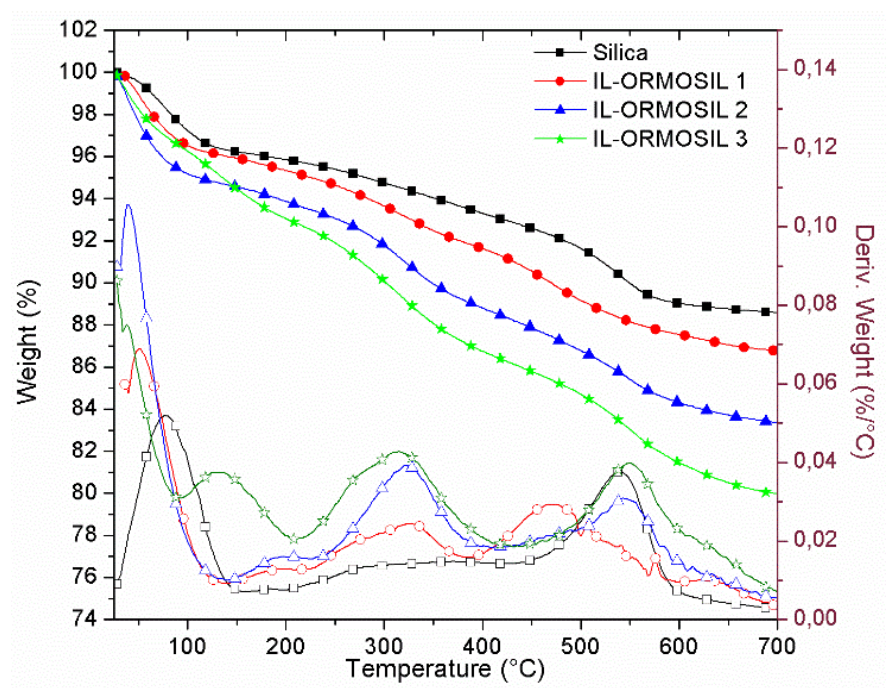

Figure 4. TGA curves of the silica, and the IL-ORMOSIL prepared with 5\% (IL- 
ORMOSIL 1), 10\% (IL-ORMOSIL 2) and 20\% (IL-ORMOSIL 3) of IL-TMOS precursor.

Table 1. Amount of IL in the IL-ORMOSIL particles estimated from TGA analysis.

\begin{tabular}{cccccccc}
\hline Sample Code & $\begin{array}{c}\text { IL/TEOS } \\
\text { molar ratio }\end{array}$ & $\begin{array}{c}\text { Mass loss } \\
\text { at } 200{ }^{\circ} \mathrm{C} \\
\text { (A) }(\%)\end{array}$ & $\begin{array}{c}\text { Mass loss } \\
\text { at } 700{ }^{\circ} \mathrm{C} \\
(\mathrm{B})(\%)\end{array}$ & $\begin{array}{c}\text { (B) }(\mathrm{A}) \\
(\%)\end{array}$ & $\begin{array}{c}\text { Estimated } \\
\text { amount of } \\
\mathrm{IL} \\
(\mathrm{wt} \%)\end{array}$ & $\begin{array}{c}\text { Incorporation } \\
\text { efficiency } \\
(\%)\end{array}$ & $\begin{array}{c}\text { Density } \\
\left(\mathrm{g} / \mathrm{cm}^{3}\right)\end{array}$ \\
\hline Silica & - & 4.0 & 11.4 & 7.4 & 0 & - & 1.98 \\
IL-ORMOSIL 1 & 0.05 & 4.7 & 13.2 & 8.5 & 2.9 & 58 & 2.01 \\
IL-ORMOSIL 2 & 0.10 & 6.1 & 16.6 & 10.5 & 8.5 & 85 & 2.02 \\
IL-ORMOSIL 3 & 0.20 & 7.0 & 20.0 & 13.0 & 13.9 & 70 & 1.99 \\
\hline
\end{tabular}

XPS spectroscopy was also used to confirm the synthesis of ORMOSILs. Figure 5 illustrates the XPS spectrum of the IL-ORMOSIL 1, where it is possible to observe the characteristic peaks with binding energies of $532.7,401.7,286$, 198 and $103.7 \mathrm{eV}$ assigned to the oxygen (O1s), nitrogen (N1s), carbon (C1s), chlorine $(\mathrm{Cl} 2 \mathrm{p})$ and silicon $(\mathrm{Si} 2 \mathrm{p})$, respectively. The presence of nitrogen, carbon and chorine confirms the presence of silylated IL covalently linked to the silica particles. 


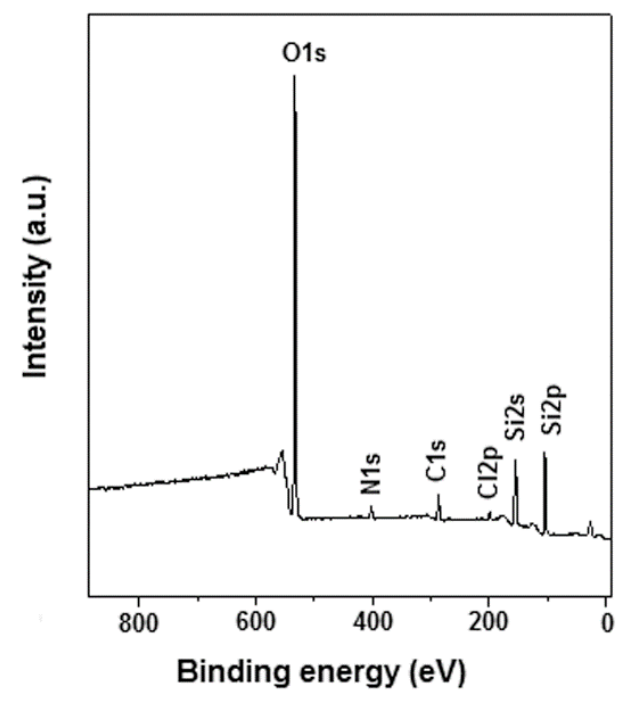

Figure 5. XPS spectrum of IL-ORMOSIL 1 (prepared with 5\% of IL-TMOS precursor).

The atomic concentration (\%) of the main elements in the different ILORMOSIL samples determined by XPS is summarized in Table 2. By comparing the intensity of $\mathrm{Si}$ and $\mathrm{N}$, it was possible to estimate the amount of the IL-TMOS attached to the silica particles around 4, 10 and 19\% for the IL-ORMOSIL 1, ILORMOSIL 2 and IL-ORMOSIL 3, respectively. This technique confirms the successful synthesis of the IL-ORMOSIL. As this technique is able to analyze only the particle surface, the amount of IL-TMOS calculated from this technique can not be used as a true amount of the IL-TMOS in the particle but should give an idea of the incorporation at the silica surface.

Table 2. Elemental composition of IL-ORMOSIL particles analyzed by XPS.

\begin{tabular}{cccc}
\hline element & IL-ORMOSIL 1 & IL-ORMOSIL 2 & IL-ORMOSIL 3 \\
\hline Si & 31.3 & 28.0 & 21.4 \\
$\mathrm{O}$ & 53.4 & 47.4 & 33.0 \\
$\mathrm{C}$ & 11.5 & 17.4 & 34.1 \\
$\mathrm{~N}$ & 2.5 & 4.4 & 7.2
\end{tabular}




\subsection{Rheological behaviour of the particle suspensions.}

The complex viscosity of the suspensions containing 15 vol. \% of particles was analyzed in the absence of an external electrical field. This test was performed in oscillatory mode using a strain sweep at $0.01-10 \%$ stain amplitude and constant frequency of $10 \mathrm{rad} / \mathrm{s}$ at $25^{\circ} \mathrm{C}$. Figure 6 illustrates the dependence of complex viscosity on the strain amplitude. The suspension containing silica particles displayed low viscosity, which did not depend on the applied strain.. The presence of the IL-ORMOSIL resulted in a substantial increase of this property. This shear thinning behavior could come from particles. The highest viscosity value was observed for the suspension containing IL-ORMOSIL 2. The suspension prepared with IL-ORMOSIL 3 (with higher amount of incorporated IL-TMOS) displayed lower viscosity than the other IL-ORMOSIL - based suspensions. This suspension was unstable and presented gross phase separation. That could explain its lower viscosity.

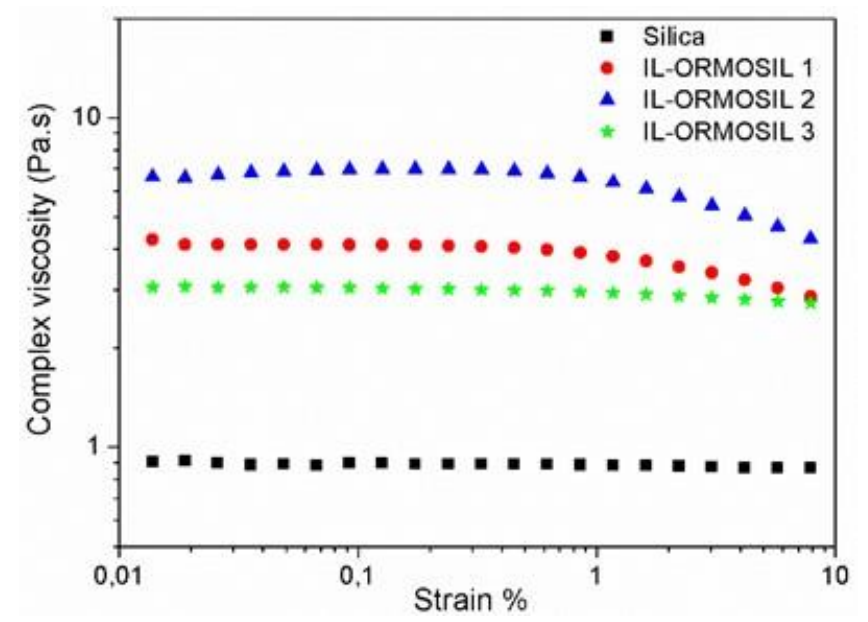

Figure 6. Complex viscosity of silica suspensions, IL-ORMOSIL 1, ILORMOSIL 2 and IL-ORMOSIL 3 in the absence of external electrical field.

\subsection{Electrorheological behavior.}

Figure 7 presents the dependence of the shear stress versus shear rate for the ERFs containing 15 vol.\% of IL-ORMOSIL particles, measured at $4 \mathrm{kV} / \mathrm{mm}$ (flow curves in the control shear stress (CSS) mode; containing 14 points with a measuring time of 60s/point). The suspension containing pure silica could not be 
measured at this electrical field magnitude. The suspensions containing ORMOSIL 1 and ORMOSIL 2 presented an increase of the shear stress at very low shear rate. The best ER response was observed for the suspension containing 15 vol.\% of IL-ORMOSIL 2. A value of dynamic shear stress corresponding to $2100 \mathrm{~Pa}$ was obtained by Bighman's Model [45], which also increased as the shear rate increases. This value is very high, as compared with other systems based on silica particles containing confined phosphonium-based ionic liquid [2627]. The IL-ORMOSIL 3 resulted in lower ER response due to the instability of the this suspension. The curve related to the suspension containing ORMOSIL 3 displayed significantly lower shear stress at lower shear rate and evean at higher shear rate, the shear stress was lower than those obtained by the other suspension. This results may be attributed to instable dispersion of this dsispersion, with visible phase separation.

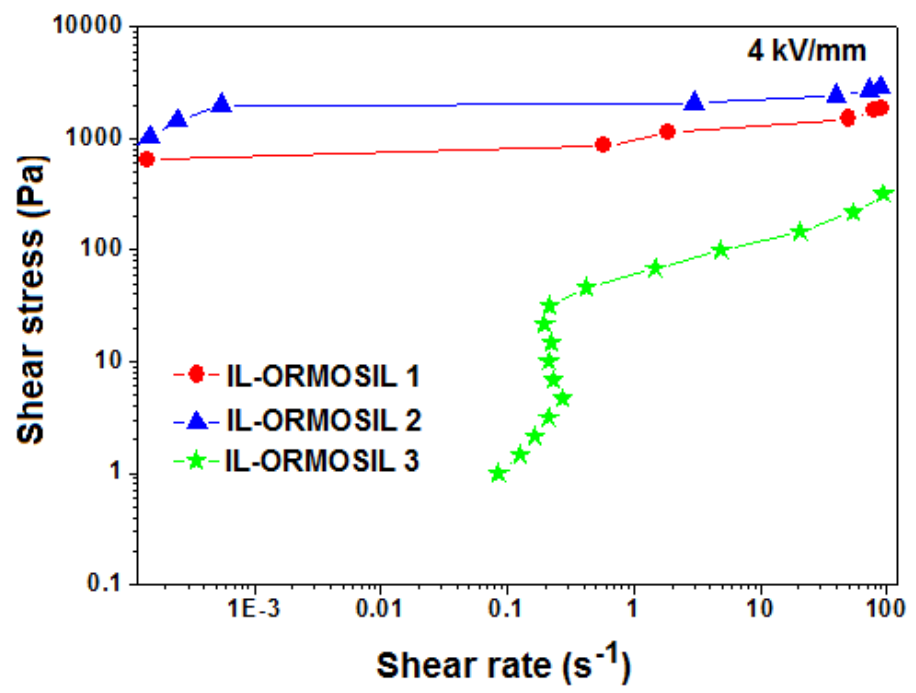

Figure 7. Flow curves (shear stress vs shear rate measured in CSS mode) of ILORMOSIL 1, 2 and 3 fluids under 4kV/mm

The ER effect of these fluids was also evaluated by applying a pulsed electric field while measuring the shear stress at a constant shear rate $\left(0.5 \mathrm{~s}^{-1}\right)$, as a function of time. The shear stress was measured under the influence of the electric field and without any applied electric field for three times (cycle off-on as a function of time) as illustrated in Figure 8. The fluid containing pure silica displayed moderate ER effect, as previously reported [27]. During the time of 
exposition at an applied electric field of $2 \mathrm{kV} / \mathrm{mm}$, the shear stress gradiently increased, indicating that the alignment of the particles was not immediate. The fluid containing $\mathrm{SiO}_{2}$ presented a very large current density with the application of a voltage corresponding to $2 \mathrm{kV} / \mathrm{mm}$. This high current density limited the application of the electric field, and when $3 \mathrm{kV} / \mathrm{mm}$ was applied the current was up to $1 \mathrm{~mA}$, (which is the limit current of generator).

The fluids containing IL-ORMOSIL resulted in significantly better ER response. With the application of an electric field of $4 \mathrm{kV} / \mathrm{mm}$, the fluid containing IL-ORMOSIL 1 provided a very rapid ER response with a shear stress of around $1000 \mathrm{~Pa}$. Similar ER behavior was achieved for the second and third cycles of applied voltage, indicating the reproducibility of the ER response. The fluid prepared with ORMOSIL 2 presented a better response, achieving shear stress as high as $2200 \mathrm{~Pa}$ under the applied electric field of $4 \mathrm{kV} / \mathrm{mm}$ and a good reproducibility for the second and third cycles. Higher amount of IL attached to the silica was not efficient for the ER behavior. The values of the current density and shear stress are summarized in Table 3. From these results, the fluid containing IL-ORMOSIL 2 seems to be the most promising material for ERF, as compared to other fluids.

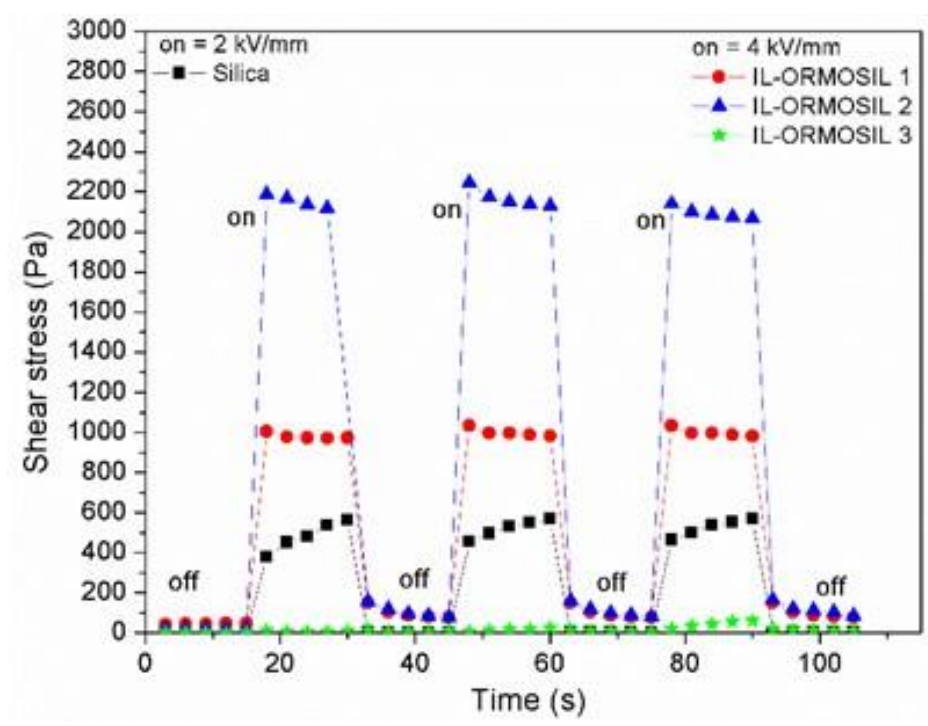

Figure 8. Shear stress as a function of time for the silica-based fluids in silicone oil, at a constant shear rate $=0.5 \mathrm{~s}^{-1}$ and under a pulsed electric field (with voltage turned on for $50 \mathrm{~s}$ and then turned off to $15 \mathrm{~s}$, three times). ER fluid of pure silica was submitted under $2 \mathrm{kV} / \mathrm{mm}$ and the other ER fluids under $4 \mathrm{kV} / \mathrm{mm}$. 
Table 3. Electrorheological feature of different fluids based on silica.

\begin{tabular}{lccc}
\hline Sample & $\begin{array}{c}\text { DC field strengh } \\
(\mathrm{kV} / \mathrm{mm})\end{array}$ & $\begin{array}{c}\text { Current density } \\
\left(\mathrm{A} / \mathrm{m}^{2}\right)\end{array}$ & Shear stress $(\mathrm{Pa})$ \\
\hline Silica & 2 & $4.10 \times 10^{-2}$ & $373-568$ \\
IL-ORMOSIL 1 & 4 & $9.69 \times 10^{-4}$ & 1000 \\
IL-ORMOSIL 2 & 4 & $1.79 \times 10^{-3}$ & 2200 \\
IL-ORMOSIL 3 & 4 & $3.16 \times 10^{-4}$ & 10 \\
\hline
\end{tabular}

The storage moduli of the ERFs at a constant frequency (10 rad/s) and different electrical fields are summarized in Table 4 for ERFs containing silica, the ILORMOSIL 1 and IL-ORMOSIL 2. All fluids presented a purely viscous behavior in the absence of an electric field, with very low value of $\mathrm{G}^{\prime}$. In the presence of an electric field, they presented a solid-like behavior $\left(G^{\prime}>G^{\prime}\right.$ ') with increased $G^{\prime}$ values as the field strength increased. This effect was more pronounced for the fluid containing IL-ORMOSIL 2, confirming the highest ER effect for this sample.

Table 4. Storage modulus of the ERFs at different DC voltages

\begin{tabular}{cccc}
\hline \multirow{2}{*}{$\begin{array}{c}\text { Electric Field } \\
\mathrm{kV} / \mathrm{mm}\end{array}$} & silica & IL-ORMOSIL 1 & IL-ORMOSIL 2 \\
\cline { 2 - 4 } & 0.057 & 0.044 & 0.055 \\
0.5 & 4 & 11 & 58 \\
1 & 21 & 66 & 148 \\
2 & 158 & 193 & 367 \\
3 & 294 & 330 & 572 \\
4 & $*$ & 449 & 811 \\
\hline
\end{tabular}

\subsection{Dielectric properties.}


The formation of the internal structure in the ERF generally depends the interfacial polarization of constitutive particles [46]. Thus, dielectric constant ( $\left.\varepsilon^{\prime}\right)$ and dielectric loss $(\varepsilon ")$ are important parameters for characterizing the ERF. Figure 9 illustrates the dielectric spectra of silica, IL-ORMOSIL 1 and ILORMOSIL 2, as a function of the frequency. The fluid containing silica did not present significant variation of $\varepsilon$ ' with the frequence, for the frequency range studied. However, both fluids containing ORMOSIL presented an increase of $\varepsilon$ ' with the decrease in frequency. This polarization effect is associated with an accumulation of charge carriers (mobile ions) at an interface between two materials (particles and a suspending liquid) [47]. The physical mechanism of ER enhanced by the presence of ILs attached on the silica particle surface may be attributed to two polarization effects, ionic and surface polarization, due to the presence of mobile ions or polar groups at the fluid-particle interface. [48]. The solid curves in Figure 9 corresponed to the fit of the experimental data by the Cole-Cole model [46]:

$$
\varepsilon^{*}=\varepsilon^{\prime}+i \varepsilon^{\prime \prime}=\varepsilon_{\infty}+\frac{\varepsilon_{0-} \varepsilon_{\infty}}{1+\left(i \omega t_{\text {relax }}\right)^{1-\alpha}}
$$

where $\varepsilon_{\infty}$ is the dielectric constant at infinite frequency $(\omega) ; \varepsilon_{0}$ is the dielectric constant of zero frequency ; $\alpha$ is an exponent related to the broadness of the relaxation time distribution; the dielectric relaxation time, $\left(t_{\text {relax }}=1 /\left(2 \pi f_{\max }\right)\right)$ is defined as the rate of interfacial polarization when an electric field is applied. Also, $\mathrm{f}_{\max }$ is the frequency at the maximum of the dielectric loss ( $\varepsilon$ "). For both ERFs, the Cole-Cole model fits relatively well to experimental data. Thus it is possible to estimate the main dielectric parameters, as summarized in Table 5.

The dielectric relaxation strength, $\Delta \varepsilon=\varepsilon_{0}-\varepsilon_{\infty}$, is higher for the IL-ORMOSIL 2 - based suspension, $(\Delta \varepsilon=36.2)$, indicating higher polarizability, due to the presence of the IL at the silica surface in higher extent. This value is significantly higher than those found in the literature for the lithium titanate nanoparticle [49] or PILs-based nanoparticles [28-29]. Also the interfacial polarization time $\left(\mathrm{t}_{\text {relax }}\right)$ is shorter for this system, indicating faster polarization response. Indeed, the relaxation time found in the present work was significantly shorter than that reported in the literature for the suspension involving lithium titanate nanoparticle 
[49]. These results are in agreement with the best ER response of the ILORMOSIL 2 - based suspension.

Table 5. Parameters of the Cole-Cole model for the IL-ORMOSIL 1 and ILORMOSIL 2 - based suspensions.

\begin{tabular}{lcccccc}
\hline Sample & $\varepsilon_{0}$ & $\varepsilon_{\infty}$ & $\Delta \varepsilon$ & $\alpha$ & $\mathrm{t}_{\text {relax }}(\mathrm{s})$ & $\mathrm{R}^{2}$ \\
\hline IL-ORMOSIL 1 & 41.5 & 7.4 & 34.1 & 0.26 & $5.9 \times 10^{-4}$ & 0.52 \\
IL-ORMOSIL 2 & 41.7 & 5.5 & 36.2 & 0.60 & $2.2 \times 10^{-5}$ & 0.96 \\
\hline
\end{tabular}
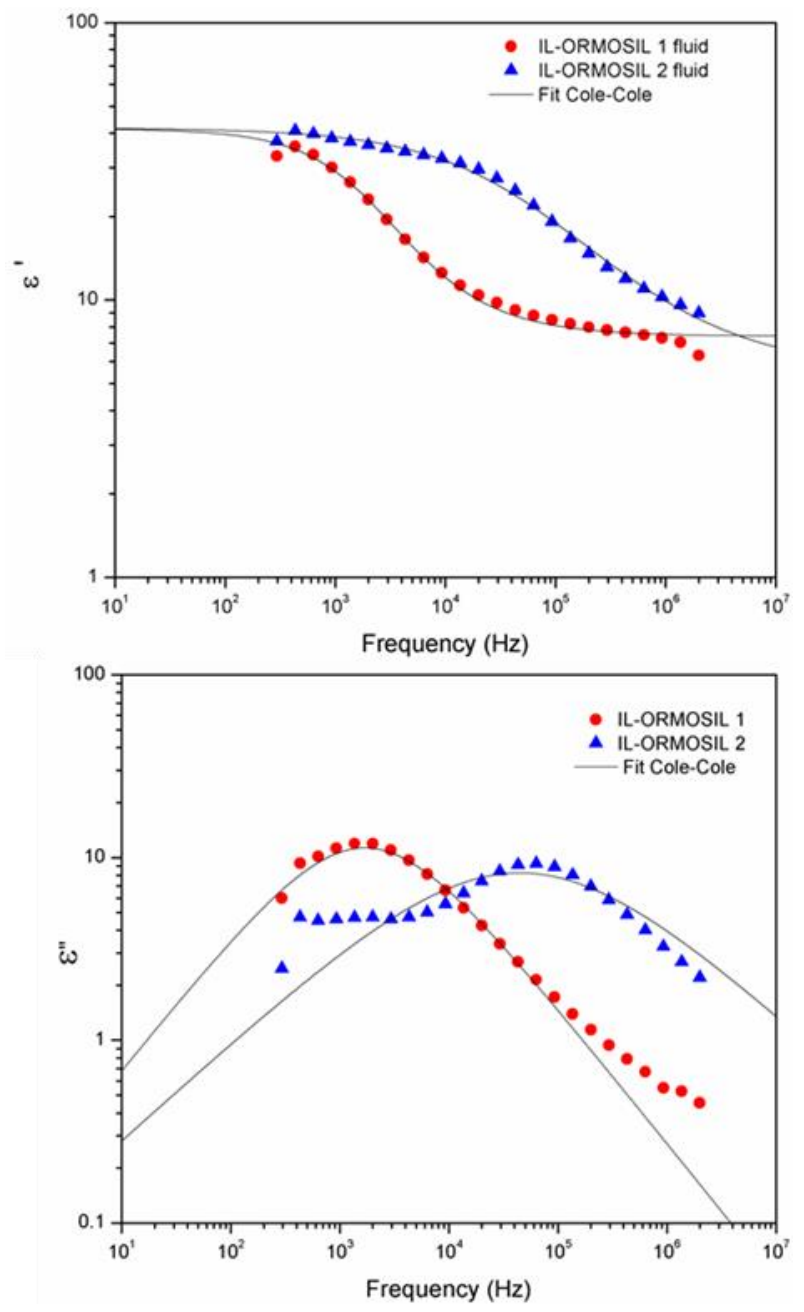

Figure 9. Dielectric constant and dielectric loss factors versus frequency for ORMOSIL 1 and ORMOSIL 2 fluids fitted by the Cole-Cole model

\section{Conclusions}


Ionogel-based organically modified silica with controlled morphology was successfully prepared by one-pot sol-gel process involving the hydrolysis/cocondensation of TEOS with silylated imidazolium - based ionic liquid (ILTMOS). Different amounts of IL-TMOS were used in the synthesis. The suspension of these organically functionalized silica bearing imidazolium-based IL covalently linked to the silica particles (IL-ORMOSIL) in silicone oil exhibited excellent electrorheological (ER) response depending on the amount of the silylated IL incorporated to the silica particles, under the applied electrical field of $4 \mathrm{kV} / \mathrm{mm}$, . Indeed, a shear stress of around $2100 \mathrm{~Pa}$ was very rapidly achieved using the suspension of IL-ORMOSIL 2 (containing around 10\% of silylated IL). Higher amount of ionic liquid in the ORMOSIL resulted in worse ER effect because of the instability of the suspension. The outstanding ER effect is attributed to the high polarizability of this suspension, high dielectric relaxation strength $(\Delta \varepsilon=36.2)$ and very low relaxation time $\left(3.5 \times 10^{-6} \mathrm{~s}\right)$, resulting in faster rate of polarization. The ER performance and dielectric properties of these ILORMOSIL particles are significantly higher than those previously reported involving sulfonated polyhedral-oligomeric sillsesquioxane particles [50], graphene oxide [46], urea-modified silica particles [13], and also the silica particles containing ionic liquid physically confined within the silica particles [26-27]. Summarizing, the results presented in this work offer new opportunities for developing smart ER fluids with outstanding, rapid and reversible response without the problem of leaching the IL during the service life.

\section{Acknowledgements}

This work was sponsored by Conselho Nacional de Desenvolvimento Científico e Tecnológico- CNPq (Grant number 303457/2013-9), and Fundação de Amparo à Pesquisa do Estado do Rio de Janeiro - FAPERJ (Grant number E26/201.183/2014). Dr. J.A. Marins also thanks the CNPq for the fellowship (PDJ

- 158856/2014-7). The authors are also thankful to Miss Amanda Garcez from Instituto de Química - UFRJ for the XPS analysis and Miss Bruna O. F. Araujo for the contribution to the experimental part.

\section{References}


[1] T. Hao, Electrorheological suspensions. Adv. Colloid Interf. Sci. 97 (2002) 135 .

[2] J.W.Kim, H.J. Choi, H.G. Lee, S.B. Choi, Damping characteristics of polyaniline-based electrorheological fluid. J. Ind. Eng. Chem. 7 (2001) 218222.

[3] C. Gehin, J. Persello, D. Charraut, B. Cabane, Electrorheological properties and microstructure of silica suspensions. J. Colloid Interf. Sci. 273 (2004) 658-667.

[4] J.A. Marins, F. Giulieri, B.G. Soares, G. Bossis, Hybrid polyaniline-coated sepiolite nanofibers for electrorheological fluid applications. Synth. Met. 185186 (2013), 9-16.

[5] Y. Otsubo, M. Sekine, S. Katayama, Electrorheological properties of silica suspensions. J. Rheol. 36 (1992) 479-496.

[6] C. Gehin, J. Persello, Effect of surface modification of colloidal silica on electrorheological properties. Int. J. Modern Physics B 16 (2002) 2494-2500.

[7] L. Rejón, F. Bautista, O. Manero, The dielectric spectrum and the electrorheological effect in suspensions of varying conductivity. Part 1. Modeling of the dielectric spectrum. Coll. Surf. A: Physicochem. Eng. Aspects 301 (2007) 63-68.

[8] S.H Kwon, S.H. Piao, H.J. Choi, Electric field-responsive mesoporous suspensions: A review. Nanomaterials 5 (2015) 2249-2267.

[9] V. Pavlinek, O. Quadrat, B. Porsch, P. Saha, Electrorheological behavior of suspensions of various surface-modified porous silica particles. Coll. Surf. A: Physicochem. Eng. Aspects 155 (1999) 241-247.

[10] A.V. Agafonov, T.A. Nefedova, O.I. Davydova, Electrorheology of suspensions of mesostructured and mesoporous silica in poly(dimethylsiloxane). Colloid J. 70 (2008) 535-540.

[11] S. Lee, J. Lee, S.H. Hwang, J. Yun, J. Jang, Enhanced electroresponsive performance of double-shell $\mathrm{SiO}_{2} / \mathrm{TiO}_{2}$ hollow nanoparticles. ACS Nano 9 (2015) 4939-4949.

[12] T. Belza, V. Pavlinek, I. Kuritka, P. Saha, Electrorheological and dielectric properties of urea/ $\mathrm{SiO}_{2}$ nanocomposite suspensions modified by $\mathrm{N}, \mathrm{N}$ dimethyl-formamide. Int. J. Modern Physics B 21 (2007) 4782-4789. 
[13] T. Belza, V. Pavlinek, P. Saha, O. Quadrat, Effect of field strength and temperature on viscoelastic properties of electrorheological suspensions of urea-modified siica particles. Coll. Surf A: Physicochem. Eng. Aspects 316 (2008) 89-94.

[14] K. Cherif, S. Moalla, S. Sassi, H. Zarrouk, Electrorheological response of modified silica suspensions. J. Eur. Ceramic Soc. 27 (2007) 1199-1202.

[15] Y.D. Liu, X.M. Quan, B.M. Lee, I.G. Kim, H.J. Choi, Fabrication of ammonium persulfate coated silica microsphere via chemical grafting and its electrorheology. J. Mater. Sci. 49 (2014) 2618-2623.

[16] J.A. Marins, K. Dahmouche, B.G. Soares, New electrorheological fluid obtained from mercaptosilsesxquioxane-modified silicate suspensions. Mater. Sci. Eng. C 33 (2013) 133-139.

[17] H.Y. Kim and H.J. Choi, Core-shell structured poly(2-ethylaniline) coated crosslinked poly(methyl methacrylate) nanoparticles by graft polymerization and their electrorheology. RSC Adv.4, (2014) 28511-28518.

[18] Y.D. Liu , K. Zhang, W.L. Zhang and H.J. Choi, Conducting Materialincorporated Electrorheological Fluids: Core-shell Structured Spheres. Aust. J. Chem. 65 (2012) 1195-1202.

[19] .A. Vioux, L. Viau, S. Volland, J. Bideau, Use of liquid ionics in sol-gel; ionogels and applications. C. R. Chimie 13 (2010) 242-255.

[20] M.V. Migliorini, R.K. Donato, M.A. Benvegnu, R.S. Gonçalves, H.S. Schrekker, Imidazolium ionic liquids as bifunctional materials (morphology controller and pre-catalyst) for the preparation of xerogel silica's. J. Sol-Gel Sci. Technol.48 (2008) 272-276.

[21] R.K. Donato, M.V. Migliorini, M.A. Benvegnú, M.P. Stracke, M.A. Gelesky, F.A. Pavan, C.M.I. Schrekker, E.V. Benvenutti, J. Dupont, H.S. Schrekker, Synthesis of silica xerogels with highly distinct morphologies in the presence of imidazolium ionic liquids. J. Sol-Gel.Sci. Technol. 49 (2009) 71-77.

[22] A. Karout, A.C. Pierre, Silica xerogels and aerogels synthesized with ionic liquids. J. Non-Cryst. Solids 353 (2007) 2900-2909.

[23] M.A. Néouze, J. Le Bideau, P. Gaveau, S. Bellayer, A. Vioux, Ionogels, new materials arising from the confinement of ionic liquids within silica-derived networks. Chem. Mater. 18 (2006) 3931-3936. 
[24] L. Viau, M.A. Neouze, C. Biolley, S. Volland, D. Brevet, P. Gaveau, P. Dieudonné, A. Galarneau, A. Vioux, Ionic liquid mediated sol-gel synthesis in the presence of water or formic acid: Which synthesis for which material? Chem. Mater. 24 (2012) 3128-3134.

[25] J. Zhang, Q. Zhang, F. Shi, S. Zhang, B. Qiao, L. Liu, Y. Ma, Y. Deng, Greatly enhanced fluorescence of dicyanamide anion based ionic liquids confined into mesoporous silica gel. Chem. Phys. Lett. 461 (2008) 229-234.

[26] J.A. Marins, B.G. Soares, A.A. Silva, M.G. Hurtado, S. Livi, Electrorheological and dielectric behavior of new ionic liquid/silica systems. J. Coll. Interf. Sci.405 (2013) 64-70.

[27] J.A. Marins, B.G. Soares, A.A. Silva, S. Livi, Silica prepared in the presence of alkylphosphonium-based ionic liquids and its performance in electrorheological fluids. RSC Adv. 4 (2014) 50925-50931.

[28] Y. Dong, J. Yin, X. Zhao, Microwave-synthesized poly(ionic liquid) particles: a new material with high electrorheological activity. J. Mater. Chem. A 2 (2014) 9812-9819.

[29] Y. Dong, J. Yin, J. Yuan, X. Zhao, Microwave-assisted synthesis and highperformance anhydrous electrorheological characteristic of monodisperse poly(ionic liquid) particles with different size of cation/anion parts. Polymer 97 (2016) 408-417.

[30] S.S. Moganty, S. Srivastava, Y. Lu, J.L. Schaefer, S.A. Rizvi, L.A. Archer, Ionic liquid-tethered nanoparticle suspensions: A novel class of ionogels. Chem. Mater. 24 (2012) 1386-1392.

[31] L. Vidal, M.L. Riekkola, A. Canals, Ionic liquid-modified materials for solidphase extraction and separation: a review. Anal. Chim. Acta 715 (2012) 19-41.

[32] S. Rostamnia, A. Hassankhani, H.G. Hossieni, B. Gholipour, H. Xin, Bronsted acidic hydrogensulfate ionic liquid immobilized SBA-15: $\{\mathrm{MPIm}]\left[\mathrm{HSO}_{4}\right] @$ SBA-15 as an environmentally friendly, metal- and halogen-free recyclable catalyst for Knoevenagel-Michael-cyclization. J. Mol. Catal. A: Chem. 395 (2014) 463-469.

[33] J. Davarpanah, P. Rezaee, S. Elahi, Synthesis and characterization of a porous acidic catalyst functionalized with an imidazole ionic liquid, and its use for synthesis of phthalazinedione and phthalazinetrione heterocyclic compounds. Res. Chem. Intermed. 41 (2015) 9903-9945. 
[34] T. Torimoto, T. Tsuda, K.I. Okazaki, S. Kuwabata, New frontiers in materials science opened by ionic liquids. Adv. Mater. 22 (2010) 11961221.

[35] O. Fontaine, A. Touidjine, M. Marechal, C. Bonhomme, F. Ribot, B. Geffro, C. Sanchez, C. Laberty-Robert, A one-pot route to prepare class II hybrid ionogel electrolytes. New J. Chem. 38 (2014) 2008-2015.

[36] A.P.A. Carvalho, B.G Soares, S. Livi, Organically modified silica (ORMOSL) bearing imidazolium-based ionic liquid prepared by hydrolysis/cocondensation of silane precursors: synthesis, characterization and use in epoxy networks. Eur. Polym. J. 83 (2016) 311-322.

[37] S. Sahoo, P. Kumar, F. Lefebvre, S.B. Halligudi, Oxidative kinetic resolution of alcohols using chiral Mn-salen complex immobilized onto ionic liquid modified silica. Appl. Catal. A. 354 (2009) 17-25.

[38] W. Stöber, A. Fink, E. Bohn, Controlled growth of monodisperse silica spheres in the micron size range. J. Coll. Interf. Sci. 26 (1968) 62-69.

[39] L. Xiang, X. Zhao, J. Yin, Micro/nano-structures montmorillonite/titania particles with high electrorheological activity. Rheol. Acta 50 (2011) 87-95.

[40] J. Yin, X. Zhao, L. Xiang, X. Xia, Z. Zhang, Enhanced electrorheology of suspensions containing sea-urchin-like hierarchical Cr-doped titania particles. Soft Matter. 5 (2009) 4687-4697.

[41] A.S. Amarasekara, O.S. Owereh, Homogeneous phase synthesis of cellulose carbamate silica hybrid materials using 1-n-butyl-3-methylimidazolium chloride ionic liquid medium. Carbohydrate Polym. 78 (2009) 635-638.

[42] N. Nagai, H. Hashimoto, FT-IR-ATR study of depth profile of $\mathrm{SiO} 2$ ultra-thin films. Appl. Surface Sci. 172 (2001) 307-311.

[43] A. Bertora, M. Castellano, E. Marsano, M. Alessi, L. Conzatti, P. Stagnaro, G. Colucci, A. Priola, A. Turturro. A new modifier for sílica in reinforcing SBR elastomers for the tyre industry. Macromol. Mater. Eng. 296 (2011) 455-464.

[44] C. Maton, N. De Vos, C.V. Stevens, Ionic liquid thermal stabilities: decomposition mechanisms and analysis tools. Chem. Soc. Rev. 42 (2013) 5963-5977.

[45] S. G. Kim, J.Y. Lim, J.H. Sung, H.J. Choi, Y. Seo. Polymer 48 (2007) 66226631 . 
[46] W.L. Zhang, Y.D. Liu, H.J. Choi, S.G. Kim, Electrorheology of graphene oxide. ACS Appl. Mater. Interfaces 4 (2012) 2267-2272.

[47] Z.M. Dang, L. Wang, Y. Yin, Q. Zhang, Q.Q. Lei, Giant dielectric permittivities in functionalized carbon-nanotube/electroactive-polymer nanocomposites. Adv. Mater. 19 (2007) 852-857.

[48] A. Krztoń-Maziopa, M. Ciszewska, J. Płocharski, Polarization processes in electrorheological fluids based on conductive polymers. Polym. Adv. Technol. 17 (2006) 37-40.

[49] T. Plachy, M. Mrlik, Z. Kozakova, P. Suly, M. Sedlacik, V. Pavlinek, I. Kuritka, The electrorheological behavior of suspensions based on molten-salt synthesized lithium titanate nanoparticles and their core-shell titanate/urea analogues. ACS Appl. Mater. Interfaces 7 (2015) 3725-2731.

[50] E.C. McIntyre, H. Yang, P.F. Green, Electrorheology of polystyrene filler/polyhedral silsesquioxane suspensions. ACS Appl. Mater. Interfaces 4 (2012) 2148-2153. 